\title{
Intracranial Calcification Associated with 3-Methylcrotonyl-CoA Carboxylase Deficiency
}

\author{
Süleyman Şahin ${ }^{a}$ Miraç Yıldırım ${ }^{a}$ Ömer Bektaşa illknur Sürücü Karab \\ Ahmet Cevdet Ceylan ${ }^{c, d}$ Serap Teber ${ }^{a}$ \\ aDepartment of Pediatric Neurology, Ankara University Faculty of Medicine, Ankara, Turkey; ${ }^{b}$ Department of \\ Pediatric Metabolism and Nutrition, Ankara University Faculty of Medicine, Ankara, Turkey; ' Department of Medical

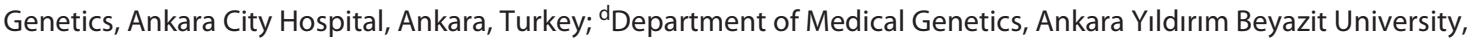 \\ Ankara, Turkey
}

\section{Established Facts}

- Patients with 3-methylcrotonyl-CoA carboxylase (3-MCC) deficiency demonstrate a variable heterogeneous clinical phenotype, ranging from neonatal onset with severe neurological disorders to asymptomatic adult forms.

- The neuroimaging findings are normal in most of the patients with 3-MCC deficiency.

- Abnormal findings such as encephalomalacia, leukodystrophy-like white matter changes, multiple cysts, corpus callosum agenesis, cerebral atrophy, necrotizing encephalopathy, periventricular white matter, and brainstem lesions are limited to just a few case reports in patients with 3-MCC deficiency.

\section{Novel Insights}

- 3-MCC deficiency may show intracranial calcifications on bilateral thalamus and periventricular white matters.

- The present cases are the first reported describing intracranial calcification in patients with 3-MCC deficiency.

\section{Keywords}

3-Methylcrotonyl-CoA carboxylase $\cdot$ Intracranial calcification · Computed tomography $\cdot M C C C 2 \cdot$ Wholeexome sequencing

\begin{abstract}
3-methylcrotonyl-CoA carboxylase (3-MCC) deficiency is the most frequent organic aciduria detected in newborn screening programs. It demonstrates a variable heterogeneous clinical phenotype, ranging from neonatal onset with severe neurological disorders to asymptomatic adult forms. Herein, we report the first 2 related cases of 3-MCC deficiency pre-
\end{abstract}

Correspondence to:

Miraç Yıldırım, miracyildirim81@ hotmail.com 
senting with intracranial calcification in the literature. A girl and a boy aged 3 years, 9 months and 4 years were included in the study. The main clinical manifestations were acquired microcephaly, global developmental delay, intractable seizures, mild feeding difficulty, and intermittent dystonic contractions. On physical and neurological examinations, their weights, heights, and head circumferences were below the 3rd percentile, they had acquired microcephaly, truncal hypotonia, upper and lower limb spasticity, hyperreflexia, positive bilateral Babinski signs, and clonus. The detailed biochemical and metabolic tests were unremarkable, except blood 3-hydroxyisovalerylcarnitine $(\mathrm{C} 5 \mathrm{OH})$ was slightly increased in case 1. Cranial computed tomography demonstrated mild cerebral and cerebellar atrophy as well as bilateral periventricular and thalamic calcifications in both cases. We identified a homozygous mutation of c.1015G >A (p. $\mathrm{V} 339 \mathrm{M}$ ) in the MCCC2gene, and the mutation was confirmed by Sanger sequencing. To the best of our knowledge, our cases are the first reported describing intracranial calcification in cases with 3-MCC deficiency. This report expands on the underlying causes of intracranial calcifications and suggests that 3-MCC deficiency may have intracranial calcifications on bilateral thalamus and periventricular white matters. If clinical findings show intracranial calcification, 3-MCC deficiency should also be kept in mind.

(c) 2021 S. Karger AG, Basel

\section{Introduction}

3-methylcrotonyl-CoA carboxylase (3-MCC) deficiency is an autosomal recessive disorder of leucine metabolism [Baumgartner et al., 2001]. The disease is caused by mutations in the $M C C C 1$ or $M C C C 2$ genes. Human MCCC1 has 19 exons and is located in chromosome region 3q25q27; MCCC2 has 17 exons and is located in chromosome region 5q12q13 [Baumgartner et al., 2001; Gallardo et al., 2001].

3-MCC deficiency is the most frequent organic aciduria detected in newborn screening programs with a prevalence ranging from $1: 2,400$ to $1: 68,000$, depending on the population, and most of the cases remain asymptomatic [Yang et al., 2015]. Patients with 3-MCC deficiency demonstrate a variable heterogeneous clinical phenotype, ranging from neonatal onset with severe neurological disorders to asymptomatic adult forms. Neurological symptoms such as global developmental delay, hypotonia, seizure, feeding difficulties, vomiting, diarrhea, involuntary movements, apnea, hyperreflexia, and spasticity have been described [Gallardo et al., 2001; Baykal et al., 2005; Ficicioglu et al., 2006]. Some patients evolve an acute metabolic crisis usually triggered by an infection or a protein-rich diet [Gallardo et al., 2001; Oude Luttikhuis et al., 2005].

The neuroimaging findings are normal in most of patients with 3-MCC deficiency. Abnormal findings have been limited to just a few case reports. The defined neuroimaging abnormalities included encephalomalacia, leukodystrophy-like white matter changes, multiple cysts, corpus callosum agenesis, cerebral atrophy, necrotizing encephalopathy, periventricular white matter, and brainstem lesions [de Kremer et al., 2002; Grünert et al., 2012; Morscher et al., 2012].

Herein, we report the first cases of 3-MCC deficiency presenting with intracranial calcification in the literature.

\section{Case Presentation}

\section{Case 1}

The 3-year, 9-month-old girl (Fig. 1; III-4) presented with microcephaly, global developmental delay, intractable seizures, mild feeding difficulty, and intermittent dystonic contractions. She was born after an uneventful pregnancy and delivery to third-degree consanguineous parents. Family history revealed the death of a sibling (a girl) who died at the age of 5 months with similar phenotypic characteristics (Fig. 1). At 6 weeks of age, seizures started as focal or generalized tonic-clonic contractions and continued despite combinations of anticonvulsant therapy such as phenobarbital, levetiracetam, topiramate, and clonazepam. She manifested an acquired microcephaly and global developmental delay in motor, social, and language skills. The motor milestones were delayed with head control at 3 years of age. She was not able to sit with or without support. She had moderate intellectual disability and required no need of assisted ventilation or permanent tube feeding. She had light tracking, but no object tracking.

On physical and neurological examinations at 3-years and 9 months, her weight, height, and head circumference were below the 3 rd percentile, she had acquired microcephaly, truncal hypotonia, upper and lower limb spasticity, hyperreflexia, positive bilateral babinski signs, and clonus. Ophthalmological, cardiac, dermatological, and hearing evaluations were normal.

The biochemical tests such as hepatic, thyroid, and renal function tests, electrolytes, levels of parathormone, and TORCH (toxoplasma, rubella, cytomegalovirus, and herpes simplex virus 2) serologies were unremarkable. Moreover, metabolic tests such as plasma, urine and cerebrospinal fluid amino acids, urine organic acids, serum ammonia level, blood gas analysis, and very longchain fatty acids tests also were unremarkable. Lysosomal enzymes such as alpha-galactosidase, beta-galactosidase, alpha-glucosidase, beta-glucosidase, sphingomyelinase were normal. 3-hydroxyisovalerylcarnitine $(\mathrm{C} 5 \mathrm{OH})$ was slightly increased in blood on tandem mass spectrometry. At 5 months of age, cranial magnetic resonance imaging (MRI) showed moderate diffuse cerebral atrophy, thinning of the corpus callosum, hypointensities in the bilateral thalamus and left centrum semiovale on T2-weighted and suscep- 


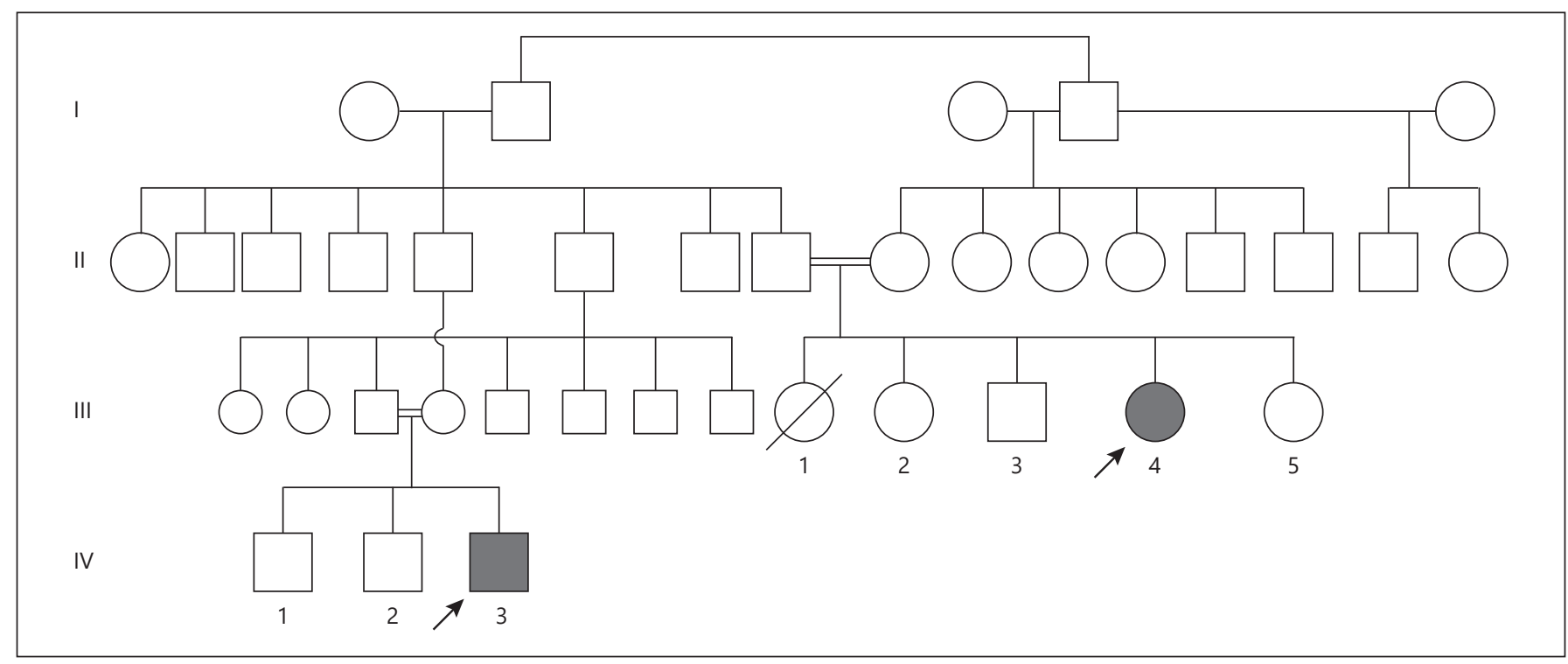

Fig. 1. Pedigree of the family carrying MCCC2 mutation-related 3-methylcrotonyl-CoA carboxylase (3-MCC) deficiency.

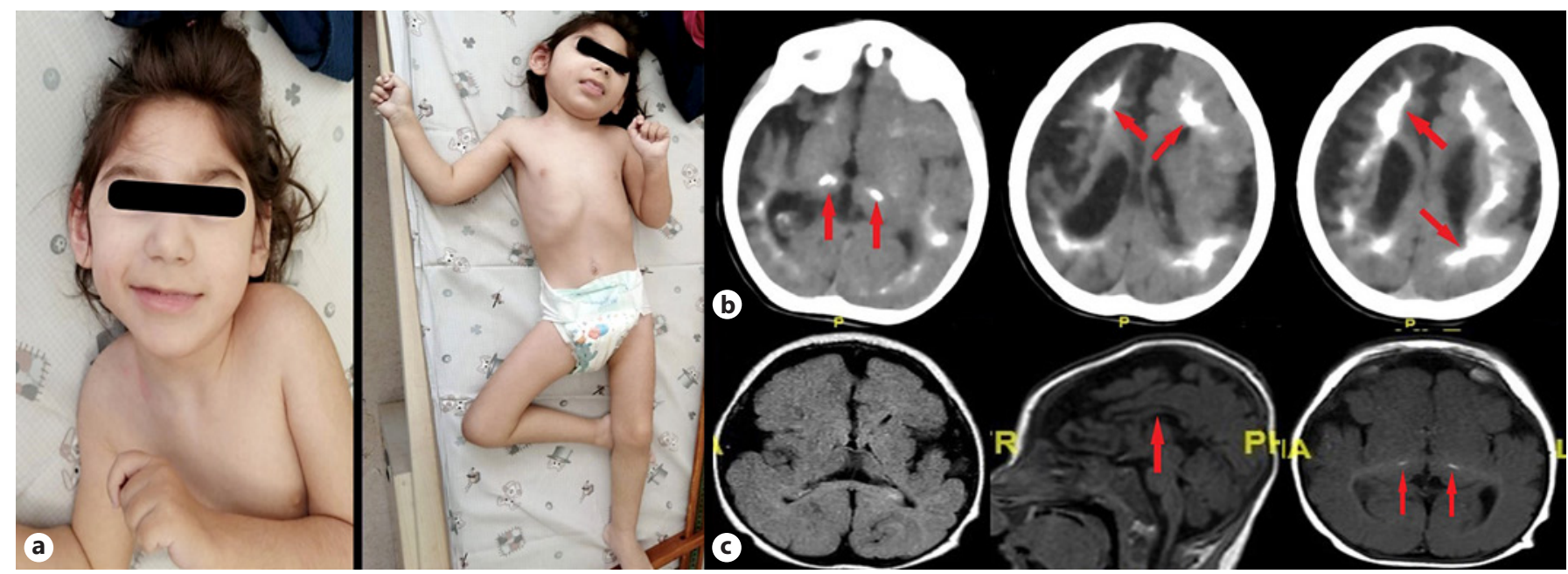

Fig. 2. a Case 1 . The girl at 3 years and 9 months showing microcephaly, global developmental delay, and intermittent dystonic contractions. b Cranial computed tomography at 3 years and 6 months of age. Ventricular system dilatation, mild cerebral atrophy, and bilateral periventricular and thalamic calcifications are shown (arrows). c Cranial magnetic resonance imaging at 5 months of age. Cerebral atrophy, abnormal sulcal pattern, thinning of the corpus callosum (arrow, middle), and hyperintensities in the bilateral thalamus on T1-weighted sequences (arrows, right).

tibility-weighted imaging (SWI) sequences, and hyperintensities in the bilateral thalamus on T1-weighted sequences. At 3 years and 6 months of age, cranial computed tomography (CT) demonstrated mild cerebral and cerebellar atrophy as well as bilateral periventricular and thalamic calcifications (Fig. 2). The EEG showed mild slow background activity and epileptic discharges with a low incidence of generalized or focal spike-wave activity.
Based on the clinical manifestations, examination, and radiological findings, we suspected the patient to have a neurometabolic disorder. Whole-exome sequencing revealed a missense mutation in the MCCC2 gene at exon 11 NM_0022132.53: c.1015G>A (p.Val339Met) using xGen Exome Research Panel v2 (Integrated DNA Technologies, Coralville, IA, USA), and the mutation was confirmed by Sanger sequencing. Her parents were both heterozygous for the same mutation. 


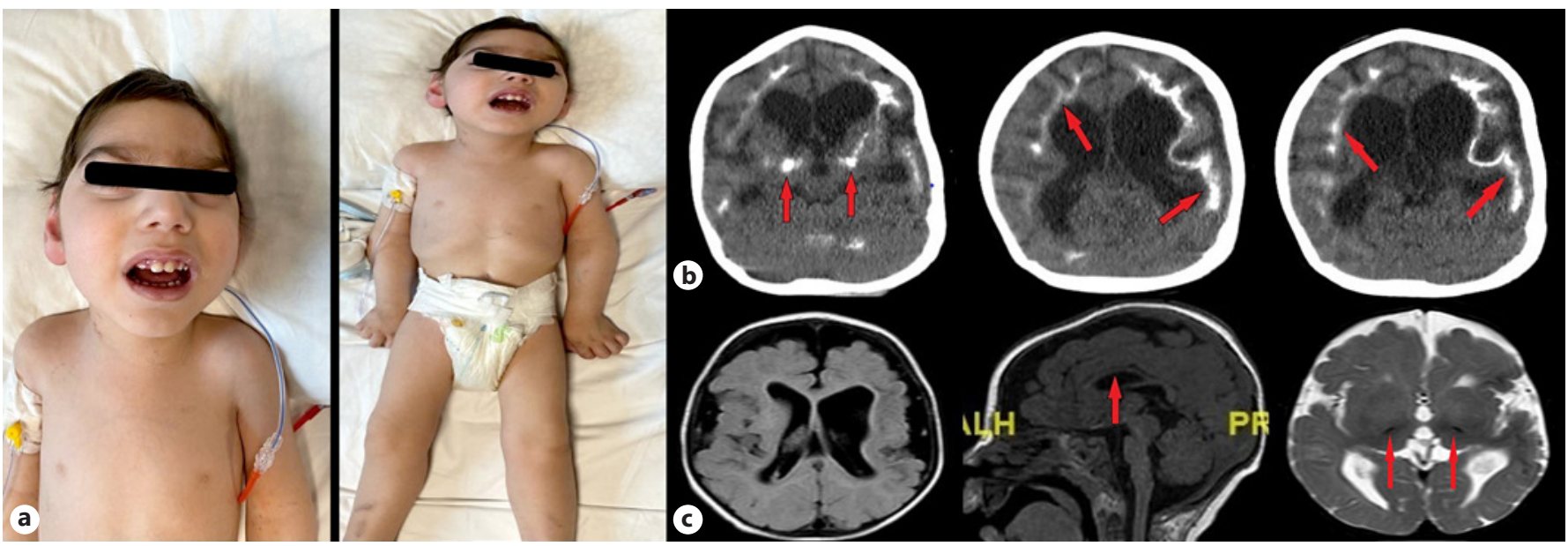

Fig. 3. a Case 2, the 4-year-old boy, showing microcephaly, global developmental delay, and intermittent dystonic contractions. b Cranial computed tomography at 3 years of age. Ventricular system dilatation, mild cerebral atrophy, and bilateral periventricular and thalamic calcifications are shown (arrows). c Cranial magnetic resonance imaging at 8 months of age. Cerebral atrophy, abnormal sulcal pattern, thinning of the corpus callosum (arrow, middle), and hypointensities in the bilateral thalamus on T2-weighted sequences (arrows, right).

Case 2

The 4-year-old boy (Fig. 1; IV-3), who is related to the first case, presented with microcephaly, global developmental delay, intractable seizures, mild feeding difficulty, and intermittent dystonic contractions. He was born after an uneventful pregnancy and delivery to third-degree consanguineous parents. At 4 months of age, seizures started as focal or generalized tonic-clonic contractions and continued despite combinations of anticonvulsant therapy such as phenobarbital, levetiracetam, topiramate, clonazepam, valproic acid, and clobazam. He manifested an acquired microcephaly and a global developmental delay in motor, social, and language skills. The motor milestones were delayed without head control. He had marked intellectual disability and required no need of assisted ventilation or permanent tube feeding. He had no light or object tracking.

On physical and neurological examinations at 4 years of age, his weight, height, and head circumference were below the 3rd percentile, he had acquired microcephaly, truncal hypotonia, upper and lower limb spasticity, hyperreflexia, positive bilateral babinski signs, and clonus. Ophthalmological, cardiac, dermatological, and hearing evaluations were normal.

The detailed biochemical and metabolic tests were unremarkable. At 8 months of age, cranial MRI showed left-hemispheric predominance of cerebral atrophy, thinning of the corpus callosum, and hypointensities in the bilateral thalamus, left predominant frontoparietal and periventricular white matter on T2-weighted and susceptibility-weighted imaging sequences. At 3 years of age, cranial CT showed mild cerebral and cerebellar atrophy, and bilateral periventricular and thalamic calcifications (Fig. 3). The EEG revealed mild slow background activity and epileptic discharges with a high incidence of generalized or focal spike-wave activity. Sanger sequencing confirmed the missense mutation in the MCCC2 gene at exon 11 NM_0022132.53: c.1015G>A (p.Val339Met). His parents were both heterozygous for the same mutation.

\section{Discussion}

We present 2 related cases with 3-MCC deficiency due to a homozygous mutation in $M C C C 2$, presenting with a severe clinical phenotype such as acquired microcephaly, global developmental delay, intractable seizures, mild feeding difficulty, and intermittent dystonic contractions. Based on the clinical manifestations, examination, laboratory findings, and genetic test results, we suggest that the present cases are typical 3-MCC deficiency disease.

To date, patients with 3-MCC deficiency were reported in a few case reports with abnormal neuroimaging findings such as encephalomalacia, leukodystrophy-like white matter changes, multiple cysts, corpus callosum agenesis, and cerebral atrophy [de Kremer et al., 2002; Grünert et al., 2012; Morscher et al., 2012]. This study reports the first cases in literature for intracranial calcification associated with 3-MCC deficiency due to a mutation in the MCCC2 gene.

Intracranial calcification consists of the brain parenchyma, choroid plexus, meninges, and vessel walls. It can be categorized into 2 groups: physiological and pathological. Physiological calcifications usually occur in various territories such as the pineal gland, choroid plexus, habenula, and dura mater [Kiroglu et al., 2010; Whitehead et al., 2015]. Pathological calcifications are catego- 
rized into 5 groups: congenital, endocrine/metabolic, infectious, vascular, and neoplastic/post-radiation therapy [Kiroglu et al., 2010]. Moreover, the locations of intracranial calcifications may be categorized into 5 main anatomical territories: midsagittal, deep gray matter, lobar, ventricular, and posterior fossa. The differential diagnosis is variable based on the location of intracranial calcifications. For example, intracranial calcifications on thalamus occur in various conditions such as Down syndrome, hypo- and hyperparathyroidism, pseudohypoparathyroidism, Fahr disease, AicardiGoutières syndrome, Krabbe disease, MELAS (mitochondrial encephalopathy, lactic acidosis, and strokelike episodes), Kearns-Sayre syndrome, Cockayne syndrome, molybdenum cofactor deficiency A, sulfite oxidase deficiency, and pseudo-TORCH syndrome 1 [Gonçalves et al., 2020]. On the other hand, intracranial calcifications on periventricular white matter occur in: infections (including cytomegalovirus, toxoplasmosis, rubella, Zika virus, post-ventriculitis), tuberous sclerosis, Aicardi-Goutières syndrome, COL4A1 mutation, juvenile Alexander disease, $\mathrm{X}$-linked adrenoleukodystrophy, pseudo-TORCH syndrome 2 , and early infantile epileptic encephalopathy-49 [Gonçalves et al., 2020]. In the present study, we investigated these possible underlying causes in both of our patients, but we determined no additional condition.

CT is the most sensitive method to demonstrate intracranial calcifications [Go and Zee, 1998]. On the other hand, they show a variable appearance on MRI. Conventional T1-weighted and T2-weighted imaging have limited sensitivity. Calcifications may appear hypo- or hyperintense on T1-weighted imaging and hypointense on T2-weighted imaging. Susceptibility-weighted imaging is a sensitive sequence for the description of hemorrhage or calcification. Both hemorrhages and calcifications indicate a low signal and often artifacts on the magnitude images [Halefoglu and Yousem, 2018; Gonçalves et al., 2020]. In the present study, we showed suspicious imaging findings on MRI for calcifications in both cases, then confirmed by CT. We suggest that the presence of similar neuroimaging findings in 2 related cases with similar clinical features strengthens the connection between the neuroimaging findings and the present disease. To the best of our knowledge, our study reports the first cases describing intracranial calcification in patients with 3-MCC deficiency.

This report expands on the underlying causes of intracranial calcifications and suggests that 3-MCC deficiency may have intracranial calcifications on bilateral thalamus and periventricular white matters. If the clinical and radiological findings in patients show intracranial calcification, 3-MCC deficiency should also be kept in mind.

\section{Acknowledgement}

We thank the patients and their families for their helpful participation in this work.

\section{Statement of Ethics}

Informed consent for genetic analysis and publication of clinical reports and photographs were obtained from the patients' parents in compliance with the national ethics regulation. There are no names or numbers indicating the patients' identity.

\section{Conflict of Interest Statement}

The authors have no conflicts of interest to declare.

\section{Funding Sources}

There was no funding for this study.

\section{Author Contributions}

S.Ş. and M.Y. wrote the manuscript and designed the figures. Ö.B. and S.T. developed the main conceptual ideas and critically reviewed the manuscript. İ.S.K. evaluated the metabolic tests and drafted the manuscript. A.C.C. carried out and evaluated the genetic tests such as whole-exome sequencing analysis, designed the pedigree and drafted the manuscript. All authors approved the final version of this manuscript.

References

Baumgartner MR, Almashanu S, Suormala T, Obie C, Cole RN, Packman S, et al. The molecular basis of human 3-methylcrotonylCoA carboxylase deficiency. J Clin Invest. 2001;107:495-504.

Baykal T, Gokcay GH, Ince Z, Dantas MF, Fowler $\mathrm{B}$, Baumgartner MR, et al. Consanguineous 3-methylcrotonyl-CoA carboxylase deficiency: early-onset necrotizing encephalopathy with lethal outcome. J Inherit Metab Dis. 2005;28:229-33.

de Kremer RD, Latini A, Suormala T, Baumgartner ER, Laróvere L, Civallero G, et al. Leukodystrophy and CSF purine abnormalities associated with isolated 3-methylcrotonyl-CoA carboxylase deficiency. Metab Brain Dis. 2002;17:13-8. 
Ficicioglu C, Payan I. 3-Methylcrotonyl-CoA carboxylase deficiency: metabolic decompensation in a noncompliant child detected through newborn screening. Pediatrics. 2006;118: 2555-6.

Gallardo ME, Desviat LR, Rodríguez JM, EsparzaGordillo J, Pérez-Cerdá C, Pérez B, et al. The molecular basis of 3-methylcrotonylglycinuria, a disorder of leucine catabolism. Am J Hum Genet. 2001;68:334-46.

Go JL, Zee CS. Unique CT imaging advantages. Hemorrhage and calcification. Neuroimaging Clin N Am. 1998;8:541-58.

Gonçalves FG, Caschera L, Teixeira SR, Viaene AN, Pinelli L, Mankad K, et al. Intracranial calcifications in childhood: Part 1. Pediatr Radiol. 2020;50:1424-47.
Grünert SC, Stucki M, Morscher RJ, Suormala T, Bürer C, Burda P, et al. 3-methylcrotonylCoA carboxylase deficiency: clinical, biochemical, enzymatic and molecular studies in 88 individuals. Orphanet J Rare Dis. 2012;7: 31.

Halefoglu AM, Yousem DM. Susceptibility weighted imaging: Clinical applications and future directions. World J Radiol. 2018;10: 30-45.

Kıroglu Y, Callı C, Karabulut N, Oncel C. Intracranial calcifications on CT. Diagn Interv Radiol. 2010;16:263-9.

Morscher RJ, Grünert SC, Bürer C, Burda P, Suormala $\mathrm{T}$, Fowler B, et al. A single mutation in MCCC1 or MCCC2 as a potential cause of positive screening for 3-methylcrotonyl-CoA carboxylase deficiency. Mol Genet Metab. 2012;105:602-6.
Oude Luttikhuis HG, Touati G, Rabier D, Williams M, Jakobs C, Saudubray JM. Severe hypoglycaemia in isolated 3-methylcrotonylCoA carboxylase deficiency; a rare, severe clinical presentation. J Inherit Metab Dis. 2005;28:1136-8.

Whitehead MT, Oh C, Raju A, Choudhri AF. Physiologic pineal region, choroid plexus, and dural calcifications in the first decade of life. AJNR Am J Neuroradiol. 2015;36:57580.

Yang L, Yang J, Zhang T, Weng C, Hong F, Tong $\mathrm{F}$, et al. Identification of eight novel mutations and transcript analysis of two splicing mutations in Chinese newborns with MCC deficiency. Clin Genet. 2015;88:484-8. 